\title{
THE OMEGA-INEQUALITY PROBLEM FOR CONCATENATION HIERARCHIES OF STAR-FREE LANGUAGES
}

\author{
J. ALMEIDA, O. KLÍMA, AND M. KUNC
}

\begin{abstract}
The problem considered in this paper is whether an inequality of $\omega$-terms is valid in a given level of a concatenation hierarchy of star-free languages. The main result shows that this problem is decidable for all (integer and half) levels of the Straubing-Thérien hierarchy.
\end{abstract}

\section{INTRODUCTION}

With the advent of computers in the 1950's, there was a surge of interest in formal languages, among which regular languages play a key role. After several examples of algebraization of questions on classes (varieties) of regular languages had been already discovered in the 1960's and early 1970's, Eilenberg described a general framework for the algebraic formulation of such questions, namely in terms of what is commonly known as Eilenberg's correspondence [14]. The algebraic structures considered by Eilenberg are finite semigroups and monoids and the classes of such structures corresponding to varieties of languages are called pseudovarieties. In general, the hope is that the algebraization will convert the membership problem for a given variety of languages in a more manageable membership problem for the corresponding pseudovariety, and indeed, the pioneering examples of instances of the correspondence before its general formulation were found with this purpose and effective application.

Eilenberg's correspondence has been extended in several directions, in particular to capture more general classes of regular languages. One such generalization was developed by Pin [20] with the aim of refining and better understanding a hierarchy of star-free languages introduced by Brzozowski and Cohen [13] and its variant considered by Straubing [29, 30] and Thérien [32]. The algebraic structures emerging in this context are finite semigroups and monoids with a compatible partial order, the relevant classes of such structures being also called pseudovarieties. While pseudovarieties of semigroups and monoids are defined by so-called pseudoidentities, which are formal equalities of members of suitable free profinite structures [27, 2], in the ordered counterpart it suffices to replace equality by formal inequality, leading to pseudoinequalities, to obtain a similar result [19, 23].

Additional motivation for investigating the Straubing-Thérien hierarchy comes from logic, specifically from finite model theory. Indeed, as has been

2010 Mathematics Subject Classification. Primary 20M05, 20M07; Secondary 20M35, $68 \mathrm{Q} 70$.

Key words and phrases. pseudovariety, relatively free profinite semigroup, ordered monoid, concatenation hierarchy, Straubing-Thérien hierarchy. 
shown by Thomas [31, the Straubing-Thérien hierarchy may be viewed as a skeleton of the hierarchy of languages defined by sentences in the first-order language with a binary predicate (for ordering positions of letters) and unary predicates for the letters of the alphabet (to denote the presence of a letter in a given position), where the complexity of sentences in prenex normal form is measured in terms of the number of quantifier alternations.

Although significant progress has been recently achieved on the membership problem for the levels of the Straubing-Thérien hierarchy [25, 26, 5], it remains an open problem whether it is decidable at all levels. A key tool that has been used in such works is the following lifting problem: to determine when a pair of elements of a finite monoid can be realized as values in the monoid of the sides of a pseudoinequality which is valid in a given level of the corresponding hierarchy of pseudovarieties. The decidability of this condition, which Place and Zeitoun [26] call the separation property, entails the decidability of the membership problem for the pseudovariety and it may be viewed as a form of hyperdecidability, namely for the inequality $x \leqslant y$, in the sense of [3]. Note that in general there are uncountably many pseudoinequalities to be considered as potential liftings of a given pair of elements of a finite monoid.

A method introduced by Steinberg and the first author [11 to approach such decidability questions consists in solving two separate problems involving an implicit signature $\sigma$ with suitable computational properties:

(1) to solve the $\sigma$-inequality problem for the pseudovariety in question;

(2) to show that, if an instance of the lifting problem has a solution, then it admits one with a pseudoinequality which is actually a $\sigma$ inequality.

Although problems such as Problem (2) tend to be very hard, they have the advantage of being abstract problems which are not of an algorithmic nature. In the aperiodic case, which is a suitable setting for the problems concerning the Straubing-Thérien hierarchy, the most frequently considered implicit signature consists of multiplication, 1 and $\omega$-power, and is also denoted $\omega$.

The main result of this paper is a solution of Problem (1) for the signature $\omega$ for every level of the Straubing-Thérien hierarchy. There are two key ingredients in the proof of this result. The first is the fact that, for a polynomially closed pseudovariety of ordered monoids $\mathrm{V}$ satisfying a pseudoinequality $u \leqslant v$, any factorization of $u$ induces a factorization of $v$ of the same length such that the inequality remains valid in $\mathrm{V}$ for factors in the same position (Section 3). The second is a syntactic analysis of $\omega$-terms that leads to a description of its factors (Section 5) and to a repetition or periodicity result (Section 6). These may be viewed as finiteness properties of $\omega$-words over the pseudovariety of all finite aperiodic monoids. For further such properties and generalizations, see [8, 15]. The combination of these results allows us to prove a sort of completeness theorem for $\omega$-inequalities valid in the Boolean-polynomial closure of any polynomially closed pseudovariety of aperiodic ordered monoids: there is a complete deductive calculus to obtain all such inequalities from a well-determined basis (Section 7). Once such a calculus has been established, one may effectively enumerate the consequences of a recursively enumerable basis. Since the $\omega$-inequalities which 
are not consequences of the basis fail in concrete finite models of the basis, decidability of the $\omega$-inequality problem follows provided both the basis and the pseudovariety of ordered monoids are recursively enumerable. Putting it all together, this yields an inductive argument that shows that all levels of the Straubing-Thérien hierarchy have decidable $\omega$-inequality problem (Section 8).

\section{Preliminaries}

The reader is referred to the standard literature on finite semigroups for general background [2, 28]. Nevertheless, we recall here some basic notions for the sake of completeness.

2.1. Pseudovarieties. A pseudovariety of monoids is a class of finite monoids that is closed under taking homomorphic images, submonoids and finite direct products. For finite ordered monoids, that is, monoids equipped with a compatible partial order, pseudovarieties are defined in the same way. A pseudovariety $\mathrm{W}$ of monoids may be identified with the pseudovariety of ordered monoids consisting of the members of $\mathrm{W}$ equipped with all possible compatible partial orders. Then, for a pseudovariety $\mathrm{V}$ of ordered monoids, the class of monoids obtained by forgetting the order of all elements of $\mathrm{V}$ and taking homomorphic images is a pseudovariety of monoids, whence it is the pseudovariety of monoids generated by $\mathrm{V}$. This is also the join of $\mathrm{V}$ with its dual, consisting of the members of $\mathrm{V}$ with the orders reversed.

The trivial pseudovariety consists of all singleton monoids. Two further examples of pseudovarieties of monoids are $\mathrm{M}$, consisting of all finite monoids, and A, consisting of all finite aperiodic monoids, that is, finite monoids all of whose subgroups are trivial.

By a pro-V monoid we mean a monoid with a compact topology for which multiplication is continuous such that distinct points may be separated by continuous homomorphisms into members of $\mathrm{V}$, these being regarded as discrete spaces. For a pseudovariety $\mathrm{V}$ of [ordered] monoids and a finite set $A$, the free pro- $\mathrm{V}$ monoid on $A$ is denoted $\bar{\Omega}_{A} \mathrm{~V}$. It may be constructed as the inverse limit of the natural projective family of $A$-generated monoids from $\mathrm{V}$. For our purposes, the relevant property is the universal property that justifies its name: every function from $A$ to a pro- $\mathrm{V}$ monoid $M$ extends uniquely to a continuous homomorphism on $\bar{\Omega}_{A} \mathrm{~V}$, as depicted in the following diagram:

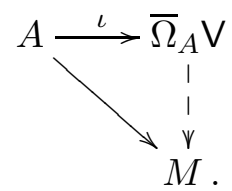

This property of $\bar{\Omega}_{A} \mathrm{~V}$ entails that each homomorphism $\varphi: A^{*} \rightarrow M$ into a pro- $\mathrm{V}$ monoid $M$ extends uniquely to a continuous homomorphism $\hat{\varphi}: \bar{\Omega}_{A} \mathrm{~V} \rightarrow$ $M$, leading to the following commutative diagram:

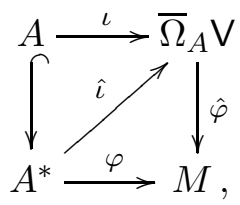


where $\hat{\iota}: A^{*} \rightarrow \bar{\Omega}_{A} \mathrm{~V}$ is the unique extension of $\iota$ to a homomorphism. In case $\alpha: A^{*} \rightarrow B^{*}$ is a homomorphism, we may also view it as a homomorphism $A^{*} \rightarrow \bar{\Omega}_{B} \mathrm{~V}$, and consider its unique extension to a continuous homomorphism $\hat{\alpha}: \bar{\Omega}_{A} \mathrm{~V} \rightarrow \bar{\Omega}_{B} \mathrm{~V}$.

Often, the natural monoid homomorphism $\hat{\iota}: A^{*} \rightarrow \bar{\Omega}_{A} \mathrm{~V}$ is an embedding, in which case we identify $A^{*}$ with its image. This is the case, for instance, for all pseudovarieties of ordered monoids containing the pseudovariety MN consisting of all finite nilpotent semigroups with an identity element adjoined, a property which holds for most nontrivial pseudovarieties of ordered monoids considered in this paper and which will therefore be used freely. Moreover, for such pseudovarieties, the topology on $A^{*}$ induced from $\bar{\Omega}_{A} \vee$ is discrete.

The elements of $\bar{\Omega}_{A} \mathrm{~V}$ are sometimes called pseudowords over $\mathrm{V}$. A pseudoinequality over $\mathrm{V}$ is a formal inequality of two pseudowords over $\mathrm{V}$; in what follows, they are simply called inequalities. The inequality $u \leqslant v$ is trivial if $u$ and $v$ coincide. For $u, v \in \bar{\Omega}_{A} \mathrm{~V}$ and $S \in \mathrm{V}$, the inequality $u \leqslant v$ is said to hold in or to be satisfied by $S$ if $\varphi(u) \leqslant \varphi(v)$ for every continuous homomorphism $\varphi: \bar{\Omega}_{A} \mathrm{~V} \rightarrow S$, and to hold in a subclass $\mathcal{C}$ of $\mathrm{V}$ if it holds in every member of $\mathcal{C}$; the pseudoidentity $u=v$ is satisfied by $\mathcal{C}$ if both inequalities $u \leqslant v$ and $v \leqslant u$ are satisfied by the members of $\mathcal{C}$. It is well known that, if an inequality $u \leqslant v$ holds in MN, that is, the pseudoidentity $u=v$ holds in $\mathrm{MN}$, and either $u$ or $v$ are words, then it is trivial.

Note that, for a pseudovariety of ordered monoids $\mathrm{V}, \bar{\Omega}_{A} \mathrm{~V}$ is an ordered monoid for the relation $\leqslant$ such that $u \leqslant v$ if and only if the inequality $u \leqslant v$ over $\mathrm{V}$ holds in $\mathrm{V}$.

For a pseudovariety $\mathrm{V}$ of ordered monoids, a language $L \subseteq A^{*}$ is said to be $\mathrm{V}$-recognizable if there exist a homomorphism $\varphi: A^{*} \rightarrow M$ into a monoid $M$ from $\mathrm{V}$ and an up-closed (that is, an order filter) subset $F$ of $M$ such that $L=\varphi^{-1}(F)$. Equivalently, the syntactic ordered monoid $\operatorname{Synt}(L)$ of $L$ belongs to $\mathrm{V}$, where $\operatorname{Synt}(L)$ is the quotient of $A^{*}$ by the congruence $\leqslant_{L} \cap \geqslant_{L}$, ordered by the partial order induced by the quasi-order $\leqslant_{L}$, where $u \leqslant_{L} v$ is defined by the following condition: for every $x, y \in A^{*}, x u y \in L$ implies $x v y \in L$. The natural homomorphism $\varphi: A^{*} \rightarrow \operatorname{Synt}(L)$ as well as its extension $\hat{\varphi}$ are both called syntactic homomorphisms. It should be noted that, in several papers by Pin and coauthors, such as 24], the syntactic order $\leqslant_{L}$ is defined to be the dual of the order considered here; see [6] for an explanation as to why our choice should be preferred.

Yet another equivalent formulation of V-recognizability of $L \subseteq A^{*}$ is that its topological closure $\bar{L}$ in $\bar{\Omega}_{A} \vee$ is open. If $\varphi: A^{*} \rightarrow M$ is a homomorphism into $M \in \mathrm{V}$ that recognizes the language $L$, then $\hat{\varphi}: \bar{\Omega}_{A} \mathrm{~V} \rightarrow M$ recognizes $\bar{L}$, in the sense that $\bar{L}=\hat{\varphi}^{-1}(\hat{\varphi}(\bar{L}))$. Note that, if $L, K \subseteq A^{*}$ are $\mathrm{V}$-recognizable languages, then the open set $\bar{L} \cap \bar{K}$ coincides with $\overline{L \cap K}$.

For pseudovariety $\mathrm{V}$ of ordered monoids, the $\mathrm{V}$-recognizable languages constitute what is known as a positive variety of languages. More precisely, a positive variety of languages is defined as a correspondence $\mathcal{V}$ associating with each finite alphabet $A$ a set $\mathcal{V}(A)$ of regular languages over $A$ that is closed under finite union, finite intersection, and the derivative operations $L \mapsto a^{-1} L=\{w: a w \in L\}, L \mapsto L a^{-1}=\{w: w a \in L\}$, and such that $L \in$ $\mathcal{V}(B)$ implies $\varphi^{-1}(L) \in \mathcal{V}(A)$ whenever $\varphi: A^{*} \rightarrow B^{*}$ is a homomorphism. 
The above defined correspondence $\mathrm{V} \mapsto \mathcal{V}$ is a bijection [20] and it is the natural analog for ordered monoids of Eilenberg's Correspondence Theorem [14, relating pseudovarieties of monoids and varieties of languages.

2.2. Polynomial closure. Given a positive variety of languages $\mathcal{V}$, its polynomial closure $\operatorname{Pol} \mathcal{V}$ is defined by letting $\operatorname{Pol} \mathcal{V}(A)$ consist of all unions of finitely many languages of the form

$$
L_{0} a_{1} L_{1} \cdots a_{n} L_{n},
$$

where the $L_{i}$ belong to $\mathcal{V}(A)$ and the $a_{i} \in A$ are letters. Note that the equality $\operatorname{Pol} \operatorname{Pol} \mathcal{V}=\operatorname{Pol} \mathcal{V}$ follows directly from the definition. We say that $\mathcal{V}$ is polynomially closed if $\mathcal{V}=\operatorname{Pol} \mathcal{V}$. It is well known that $\operatorname{Pol} \mathcal{V}$ is a positive variety of languages whenever $\mathcal{V}$ is a variety of languages. Therefore, for the pseudovariety $\mathrm{V}$ of monoids corresponding to a variety of languages $\mathcal{V}$, we denote by Pol $\vee$ the pseudovariety of ordered monoids corresponding to the positive variety of languages $\mathrm{Pol} \mathcal{V}$. A pseudovariety of ordered monoids corresponding to a polynomially closed positive variety of languages is also called polynomially closed. Let us remark that PolV is not defined for a pseudovariety of ordered monoids $\mathrm{V}$ in general.

By a result of Pin and Weil [24, Theorem 5.9], if $\mathrm{V}$ is a pseudovariety of monoids, then Pol $\vee$ may be described as the Mal'cev product $\llbracket x^{\omega} \leqslant$ $x^{\omega} y x^{\omega} \rrbracket \mathbb{O} \mathrm{V}$. Combining with a general basis theorem for Mal'cev products, also due to Pin and Weil [22], we obtain a basis of inequalities for Pol V (see [21, Proposition 7.4]).

Proposition 2.1. Let $\vee$ be a pseudovariety of monoids. Then, Pol $\vee$ is defined by all inequalities of the form $u^{\omega} \leqslant u^{\omega} v u^{\omega}$, with $u, v \in \bar{\Omega}_{A} \mathrm{M}$, such that the pseudoidentities $u=v=v^{2}$ hold in $\mathrm{V}$.

Also of interest is to consider the Boolean-polynomial closure of a pseudovariety $\mathrm{V}$ of ordered monoids, denoted Pol B V. This means taking first the pseudovariety of monoids generated by $\mathrm{V}$, which recognizes precisely the languages over a finite alphabet $A$ that are Boolean combinations of languages recognized by $\mathrm{V}$, and then taking the polynomial closure of that pseudovariety of monoids. The following basis of inequalities for Pol B V can be found in [5].

Proposition 2.2. Let $\mathrm{W}$ be a pseudovariety of monoids and let $\mathrm{V}=\mathrm{Pol} \mathrm{W}$. Then, Pol B V is defined by all inequalities of the form $u^{\omega+1} \leqslant u^{\omega} v u^{\omega}$, with $u, v \in \bar{\Omega}_{A} \mathrm{M}$, such that the inequality $v \leqslant u$ holds in $\mathrm{V}$.

The concatenation hierarchy based on a pseudovariety of monoids $\mathrm{V}$ is the sequence starting at $\mathrm{V}$ that alternates polynomial closure with forgetting order plus taking homomorphic images; the pseudovarieties of monoids obtained by forgetting order plus taking homomorphic images are called levels of the hierarchy, while the pseudovarieties of ordered monoids obtained by the polynomial closure are called half levels. The concatenation hierarchy based on the trivial pseudovariety is known as the Straubing-Thérien hierarchy. 
2.3. Validity of inequalities. The following lemma gives two alternative characterizations of when an inequality holds in a syntactic ordered monoid.

Lemma 2.3. Let $A$ and $B$ be finite alphabets, $L \subseteq B^{*}$ be a regular language, and $u, v \in \bar{\Omega}_{A} \mathrm{M}$ be pseudowords. The following conditions are equivalent:

(1) the inequality $u \leqslant v$ holds in the syntactic ordered monoid Synt $(L)$;

(2) for every homomorphism $\alpha: A^{*} \rightarrow B^{*}$ and all words $x, y \in B^{*}$, $x \hat{\alpha}(u) y \in \bar{L}$ implies $x \hat{\alpha}(v) y \in \bar{L}$;

(3) for every homomorphism $\alpha: A^{*} \rightarrow B^{*}$ and all pseudowords $x, y \in$ $\bar{\Omega}_{B} \mathrm{M}, x \hat{\alpha}(u) y \in \bar{L}$ implies $x \hat{\alpha}(v) y \in \bar{L}$.

Proof. Let $\varphi: \bar{\Omega}_{B} \mathrm{M} \rightarrow \operatorname{Synt}(L)$ be the syntactic homomorphism.

(11) $\Rightarrow$ (3) Let $\alpha: A^{*} \rightarrow B^{*}$ be a homomorphism and suppose that $x, y \in$ $\bar{\Omega}_{B} \mathrm{M}$ are such that $x \hat{\alpha}(u) y \in \bar{L}$. Applying $\varphi$ and taking into account that $\varphi(\bar{L})=\varphi(L)$, we obtain the relation $\varphi(x \hat{\alpha}(u) y) \in \varphi(L)$. Recall that $\varphi(L)$ is an order filter in $\operatorname{Synt}(L)$. Since the order in $\operatorname{Synt}(L)$ is stable and $\varphi \hat{\alpha}(u) \leqslant$ $\varphi \hat{\alpha}(v)$ by (1), it follows that $\varphi(x \hat{\alpha}(v) y) \in \varphi(L)$. Since $\varphi$ recognizes $\bar{L}$, we deduce that $x \hat{\alpha}(v) y \in \bar{L}$, as required.

The implication (3) $\Rightarrow(2)$ is trivial.

(2) $\Rightarrow$ (11) Let $\psi: \bar{\Omega}_{A} \mathrm{M} \rightarrow \operatorname{Synt}(L)$ be an arbitrary continuous homomorphism. We need to show that $\psi(u) \leqslant \psi(v)$. Choose a homomorphism $\alpha: A^{*} \rightarrow B^{*}$ such that $\psi=\varphi \circ \hat{\alpha}$. By definition of the syntactic order and since $\left.\varphi\right|_{B^{*}}$ recognizes $L$, the preceding inequality is equivalent to the property that, for all $p, q \in \operatorname{Synt}(L), p \psi(u) q \in \varphi(L)$ implies $p \psi(v) q \in \varphi(L)$. To establish this property, note first that, since $\left.\varphi\right|_{B^{*}}$ is onto, given $p, q \in \operatorname{Synt}(L)$ such that $p \psi(u) q \in \varphi(L)$, there exist $x, y \in B^{*}$ such that $\varphi(x)=p$ and $\varphi(y)=q$. Since $\varphi$ recognizes $\bar{L}$, we deduce that $x \hat{\alpha}(u) y \in \bar{L}$. By (2), it follows that $x \hat{\alpha}(v) y \in \bar{L}$, and so indeed $p \psi(v) q \in \varphi(L)$, as claimed.

Lemma 2.3 serves to establish a simple profinite characterization of when an inequality holds in a pseudovariety of ordered monoids.

Proposition 2.4. Let $\bigvee$ be a pseudovariety of ordered monoids and let $u, v \in$ $\bar{\Omega}_{A} \mathrm{M}$. The following conditions are equivalent:

(1) the inequality $u \leqslant v$ holds in $\mathrm{V}$;

(2) whenever $L \subseteq A^{*}$ is a $\mathrm{V}$-recognizable language, $u \in \bar{L}$ implies $v \in \bar{L}$.

Proof. (11) $\Rightarrow$ (2) Suppose that (1) holds and let $L \subseteq A^{*}$ be a $V$-recognizable language such that $u \in \bar{L}$. By assumption, $\operatorname{Synt}(L)$ satisfies the inequality $u \leqslant v$. By Lemma 2.3(2), taking $x=y=1$ and $\alpha$ to be the identity mapping, we deduce from $u \in \bar{L}$ that $v \in \bar{L}$, as required.

(2) $\Rightarrow$ (11) Since $V$ is generated by the syntactic ordered monoids $\operatorname{Synt}(K)$ in $\mathrm{V}$ of regular languages $K$, it suffices to show that, for every $\mathrm{V}$-recognizable language $K \subseteq B^{*}$, the ordered monoid $\operatorname{Synt}(K)$ satisfies the inequality $u \leqslant$ $v$. For this purpose, we establish the condition (2) of Lemma 2.3. Thus, we should show that, for every homomorphism $\alpha: A^{*} \rightarrow B^{*}$ and all words $x, y \in B^{*}, x \hat{\alpha}(u) y \in \bar{K}$ implies $x \hat{\alpha}(v) y \in \bar{K}$.

We claim that, for $w \in \bar{\Omega}_{A} \mathrm{M}, x \hat{\alpha}(w) y \in \bar{K}$ is equivalent to $w \in \bar{L}$, where $L=\alpha^{-1}\left(x^{-1} K y^{-1}\right)$. Suppose first that $x \hat{\alpha}(w) y \in \bar{K}$ and let $w_{n}$ be a sequence of words of $A^{*}$ converging to $w$. Since $\bar{K}$ is an open set 
such that $\bar{K} \cap B^{*}=K$ (cf. [2, Theorem 3.6.1]) and the limit $x \hat{\alpha}(w) y$ of the sequence $x \alpha\left(w_{n}\right) y$ belongs to $\bar{K}$, passing to a subsequence if necessary, we may assume that all terms in the sequence belong to $K$. Then every $w_{n}$ belongs to $L$, so $w \in \bar{L}$. The converse follows from the continuity of both $\hat{\alpha}$ and multiplication.

It remains to apply the hypothesis (2) to the language $L=\alpha^{-1}\left(x^{-1} K y^{-1}\right)$, which is $\mathrm{V}$-recognizable because the class of all V-recognizable languages constitutes a positive variety of languages.

\section{LIFTING FACTORIZATIONS}

The next result shows that factorizations may be lifted along inequalities. This property plays a key role in the sequel. The proof uses nets in compact spaces, which is a classical tool in Topology (see, for instance [33]).

Theorem 3.1. Let $\mathrm{V}$ be a polynomially closed pseudovariety of ordered monoids and let $u, v \in \bar{\Omega}_{A} \mathrm{M}$. If the inequality $u \leqslant v$ holds in $\mathrm{V}$ then, for every factorization $u=u_{0} a u_{1}$ with $a \in A$, there is a factorization $v=v_{0} a v_{1}$ such that each inequality $u_{i} \leqslant v_{i}$ holds in $\mathrm{V}(i=0,1)$.

Proof. Let $u=u_{0} a u_{1}$ be an arbitrary factorization with $a \in A$. Let $I$ be the set of all pairs $\left(L_{0}, L_{1}\right)$ of $\mathrm{V}$-recognizable languages $L_{i} \subseteq A^{*}$ such that $u_{i} \in \overline{L_{i}}$ $(i=0,1)$. We consider on $I$ the partial order defined by $\left(L_{0}, L_{1}\right) \leqslant\left(K_{0}, K_{1}\right)$ if $K_{i} \subseteq L_{i}(i=0,1)$. Since the positive variety of languages corresponding to $\mathrm{V}$ is closed under intersection, the above partial order on the set $I$ is upper directed. For each $\left(L_{0}, L_{1}\right) \in I$, since $u=u_{0} a u_{1} \in \overline{L_{0}} a \overline{L_{1}}=\overline{L_{0} a L_{1}}$ and the language $L_{0} a L_{1}$ is $\mathrm{V}$-recognizable because $\mathrm{V}$ is polynomially closed, it follows from Proposition 2.4 that $v \in \overline{L_{0}} a \overline{L_{1}}$, and so we may choose a factorization $v=v_{0}^{\left(L_{0}, L_{1}\right)} a v_{1}^{\left(L_{0}, L_{1}\right)}$ such that each $v_{i}^{\left(L_{0}, L_{1}\right)}$ belongs to $\overline{L_{i}}(i=0,1)$.

The mapping $\eta: I \rightarrow\left(\bar{\Omega}_{A} \mathrm{M}\right)^{2}$ defined by $\eta\left(L_{0}, L_{1}\right)=\left(v_{0}^{\left(L_{0}, L_{1}\right)}, v_{1}^{\left(L_{0}, L_{1}\right)}\right)$ may be viewed as a net in the compact product space $\left(\bar{\Omega}_{A} \mathrm{M}\right)^{2}$. Hence, there is a convergent subnet $\eta \circ \lambda$ determined by a mapping $\lambda: J \rightarrow I$ from another upper directed set $J$ into $I$ such that, for every $\left(L_{0}, L_{1}\right) \in I$ there is some $j \in J$ such that $\left(L_{0}, L_{1}\right) \leqslant \lambda(j)$. Let $\left(v_{0}, v_{1}\right)$ be the limit of the subnet $\eta \circ \lambda$. By continuity of multiplication, since $v=v_{0}^{\lambda(j)} a v_{1}^{\lambda(j)}$ for every $j \in J$, it follows that $v=v_{0} a v_{1}$.

We claim that each inequality $u_{i} \leqslant v_{i}$ holds in $\mathrm{V}(i=0,1)$. To establish these inequalities, we apply again Proposition 2.4 it suffices to show that, for all pairs of $\mathrm{V}$-recognizable languages $L_{i} \subseteq A^{*}$ such that $u_{i} \in \overline{L_{i}}(i=0,1)$, we have $v_{i} \in \overline{L_{i}}(i=0,1)$. Let $j_{0} \in J$ be such that $\left(L_{0}, L_{1}\right) \leqslant \lambda\left(j_{0}\right)$. Now, for every $i \in\{0,1\}$ and $j \in J$ such that $j \geqslant j_{0}$, the pseudoword $v_{i}^{\lambda(j)}$ belongs to the closure of the $i$ th component of $\lambda(j)$, which is contained in $\overline{L_{i}}$. Hence $v_{i}^{\lambda_{(j)}} \in \overline{L_{i}}$ for every $j \geqslant j_{0}$, which implies that the limit $v_{i}$ also belongs to the closed set $\overline{L_{i}}$, as claimed.

Most of the time, it will be inconvenient to keep referring to the letter $a$ in the factorizations to be lifted along inequalities considered in Theorem 3.1 . The following result avoids it and further extends the lifting to an arbitrary number of factors. 
Corollary 3.2. Let $\bigvee$ be a polynomially closed pseudovariety of ordered monoids and let $u, v \in \bar{\Omega}_{A} \mathrm{M}$. If the inequality $u \leqslant v$ holds in $\mathrm{V}$ then, for every factorization $u=u_{1} \cdots u_{n}$, there is a factorization $v=v_{1} \cdots v_{n}$ such that each inequality $u_{i} \leqslant v_{i}$ holds in $\mathrm{V}(i=1, \ldots, n)$.

Proof. Proceeding by induction, it suffices to consider the case $n=2$. So, suppose that the inequality $u \leqslant v$ holds in $\mathrm{V}$ and consider a factorization $u=u_{1} u_{2}$. If $u_{2}=1$, then the factorization $v=v \cdot 1$ has the required properties. Otherwise, taking into account that $u_{2}$ is the limit of a sequence of nonempty words, a standard compactness argument shows that there is a factorization $u_{2}=a u^{\prime}$, where $a$ is some letter from $A$. We may then apply Theorem 3.1 to lift the factorization $u=u_{1} a u^{\prime}$ along the inequality $u \leqslant v$ to a factorization $v=v_{1} a v^{\prime}$ such that the inequalities $u_{1} \leqslant v_{1}$ and $u^{\prime} \leqslant v^{\prime}$ hold in $\mathrm{V}$. It remains to take $v_{2}=a v^{\prime}$ and observe that the inequality $u_{2} \leqslant v_{2}$ also holds in $\mathrm{V}$.

\section{OMEGA-INEQUALITIES}

It is well known that elements of the free profinite monoid $\bar{\Omega}_{A} \mathrm{M}$ may be viewed as operations with a natural interpretation on each profinite monoid in such a way that the interpretation is preserved under continuous homomorphisms (see, for instance, [4]). More precisely, each $w \in \bar{\Omega}_{A} \mathrm{M}$ defines an $A$-ary operation symbol which is naturally interpreted in a profinite monoid $M$ as the operation $w_{M}: M^{A} \rightarrow M$ that maps each function $\varphi: A \rightarrow M$ to $\hat{\varphi}(w)$. By an implicit signature, we mean a set of such operation symbols including the binary multiplication and the (nullary) identity element, that is, the standard signature for working with monoids. For an implicit signature $\sigma$, each profinite monoid $M$ has thus a natural structure of a $\sigma$-algebra by interpreting each operation symbol naturally. It is a simple exercise to show that, for a pseudovariety $\mathrm{V}$ of ordered monoids, the $\sigma$ subalgebra of $\bar{\Omega}_{A} \mathrm{~V}$ generated by $A$, denoted by $\Omega_{A}^{\sigma} \mathrm{V}$, is a $\mathrm{V}$-free $\sigma$-algebra on $A$. Elements of $\Omega_{A}^{\sigma} \mathrm{V}$ will be called $\sigma$-words (over $\mathrm{V}$ ).

The absolutely free $\sigma$-algebra on a generating set $A$ is the term $\sigma$-algebra $T_{\sigma}(A)$. The members of $T_{\sigma}(A)$ are obtained recursively from the elements of $A$ by formally applying successively an operation from $\sigma$. As is standard, they may be visualized as finite rooted trees in which the leaves are labeled by members of $A$ or the constant 1 and the non-leaf nodes are labeled by elements of $\sigma$; for each non-leaf node with operation $w$ the sons are written in the order they are taken as arguments of the operation $w$. A node is called a right descendant whenever it is the second son of a node labeled by a binary operation.

Such construction and representation are unique for each $\sigma$-term $t$. For a pseudovariety $\mathrm{V}$ of ordered monoids, there is a natural homomorphism of $\sigma$-algebras $\theta: T_{\sigma}(A) \rightarrow \Omega_{A}^{\sigma} \mathrm{V}$ mapping each free generator to itself. For a $\sigma$-word $u$ over $\mathrm{V}$, a $\sigma$-term in $\theta^{-1}(u)$ is said to represent $u$.

We will be concerned with the signature $\omega$ consisting of binary multiplication and the usual $\omega$-power, whose natural interpretation on a profinite monoid $M$ maps each element $s$ to the unique idempotent in the closed subsemigroup of $M$ generated by $s$. As an example, consider the $\omega$-word $\left(a^{2} b^{\omega}\right)^{\omega} a b^{\omega}$. One of its $\omega$-term representations is described by the tree in 
Figure 1. Note that the tree has four right descendants, which are graphically indicated by the lower end of an edge going down from a node towards the right.

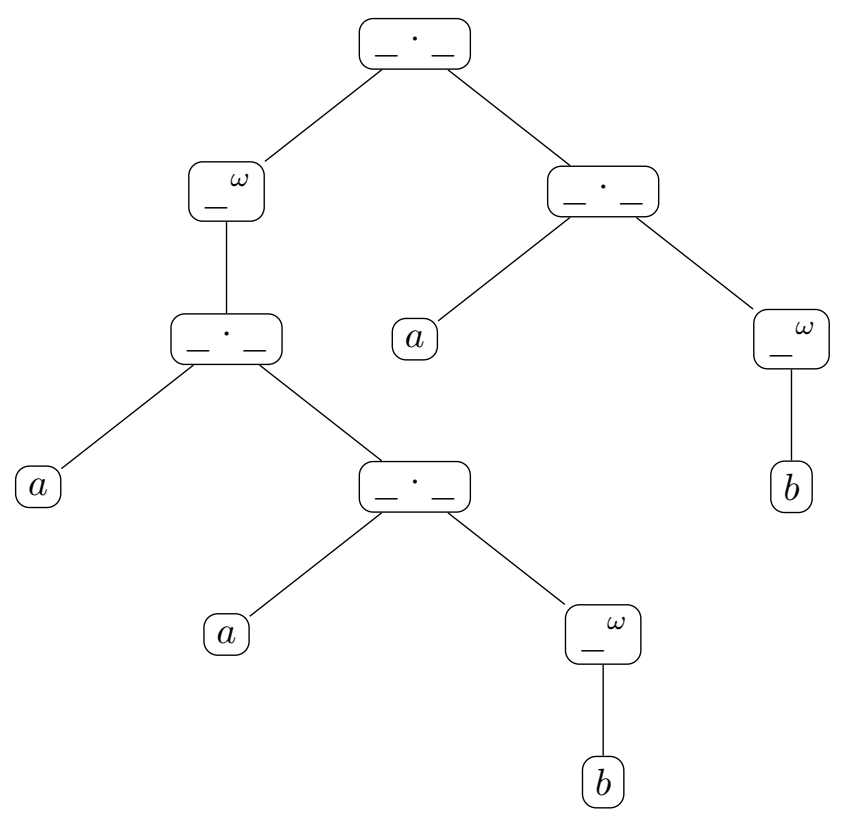

Figure 1. A tree representing the $\omega$-word $\left(a^{2} b^{\omega}\right)^{\omega} a b^{\omega}$.

By the $\omega$-inequality problem for a pseudovariety $\mathrm{V}$ of ordered monoids we mean the problem that takes as input a pair $(u, v)$ of $\omega$-terms and asks whether the inequality $u \leqslant v$ is valid in $\mathrm{V}$. Decidability of this problem amounts to being able to algorithmically calculate in the ordered monoid $\Omega_{A}^{\omega} \mathrm{V}$. Replacing inequalities by equalities, one may analogously define the corresponding notions such as decidability of the $\omega$-equality problem.

Our aim is to show that the $\omega$-inequality problem is decidable for all levels of the Straubing-Thérien hierarchy of aperiodic monoids. Rather than trying to construct efficient algorithms for such an infinite class of problems, we concentrate on "theoretical decidability", proving simply that both the instances of the problem with positive solution and those with negative solution may be recursively enumerated. For the purpose of enumerating inequalities with certain properties, it is convenient to consider only finite alphabets contained in a fixed countable set of variables, which we do from hereon without further mention.

We say that a pseudovariety of ordered monoids $\mathrm{V}$ is recursively enumerable if there is a Turing machine that successively produces as outputs precisely the elements of $\mathrm{V}$, up to isomorphism, and nothing else. Equivalently, the corresponding positive variety of languages is recursively enumerable.

Proposition 4.1. Let $\mathrm{V}$ be a recursively enumerable pseudovariety of ordered monoids. Then the $\omega$-inequality problem for $\mathrm{V}$ is co-recursively enumerable.

Proof. There is a Turing machine that successively enumerates all pairs consisting of a member $M$ of $\mathrm{V}$, up to isomorphism, and an $\omega$-inequality $u \leqslant v$, 
and nothing else. Note that the $\omega$-power of an element $s$ is computable in $M$ : for instance, one may compute it as $s^{\omega}=s^{n !}$, where $n=|M|$. Hence, one may effectively test for each such pair $(M, u \leqslant v)$ whether $M$ satisfies $u \leqslant v$ and output the pair $u \leqslant v$ in the negative case. This way, we recursively enumerate precisely the inputs for the $\omega$-inequality problem with negative output.

We are thus left with enumerating the positive cases of the $\omega$-inequality problem for suitable pseudovarieties of ordered monoids $\mathrm{V}$, a task that is accomplished in Theorem 8.2 . We are actually going to deal with inequalities of $\omega$-words from $\Omega_{A}^{\omega} \mathrm{A}$, instead of inequalities of $\omega$-terms, in order to be able to employ known properties of the monoids $\Omega_{A}^{\omega} A$. With this aim, we generalize the $\omega$-inequality problem from $\omega$-terms to $\omega$-words in a given pseudovariety. Let $\mathrm{W}$ be a pseudovariety of monoids with decidable $\omega$-equality problem, that is, such that calculations in the ordered monoids $\Omega_{A}^{\omega} \mathrm{W}$ can be performed algorithmically. By the $\omega$-inequality problem over $\mathrm{W}$ for a pseudovariety $\mathrm{V}$ of ordered monoids contained in $\mathrm{W}$ we mean the problem that takes as input a pair $(u, v)$ of $\omega$-words from $\Omega_{A}^{\omega} \mathrm{W}$ and asks whether the inequality $u \leqslant v$ is valid in $\mathrm{V}$. The following result allows us to deal only with such restricted $\omega$-inequality problems, when convenient.

Proposition 4.2. Let $\mathrm{W}$ be a pseudovariety of monoids with decidable $\omega$ equality problem and let $\mathrm{V}$ be a pseudovariety of ordered monoids contained in $\mathrm{W}$ such that the $\omega$-inequality problem for $\mathrm{V}$ over $\mathrm{W}$ is recursively enumerable. Then, the $\omega$-inequality problem for $\mathrm{V}$ is recursively enumerable.

Proof. Let $\pi: \bar{\Omega}_{A} \mathrm{M} \rightarrow \bar{\Omega}_{A} \mathrm{~W}$ be the unique continuous homomorphism mapping each generator to itself. By assumption, there is a Turing machine enumerating the quadruples $(u, v, w, z)$, where $u, v \in \Omega_{A}^{\omega} \mathrm{W}$ and $w, z \in \Omega_{A}^{\omega} \mathrm{M}$ are such that $u \leqslant v$ holds in $\mathrm{V}$. Using the decidability of the $\omega$-equality problem for $\mathrm{W}$, for each such quadruple, algorithmically calculate $\pi(u)$ and $\pi(v)$, and check whether they are equal to $w$ and $z$, respectively. In the affirmative case, output the $\omega$-inequality $w \leqslant z$. The resulting Turing machine recursively enumerates the positive cases of the $\omega$-inequality problem for $\mathrm{V}$.

\section{EQUIDIVISIBILITY AND FACTORIALITY}

We recall here the notion of equidivisibility, which was introduced in [18]. A semigroup $S$ is said to be equidivisible if, for all $s, t, u, v \in S$ such that $s t=u v$, there is $w \in S^{1}$ such that either $s=u w$ and $w t=v$, or $s w=u$ and $t=w v$. A pseudovariety of monoids $\mathrm{V}$ is equidivisible if $\bar{\Omega}_{A} \mathrm{~V}$ is equidivisible for every finite set $A$. A pseudovariety of monoids $\mathrm{V}$ is said to be closed under concatenation if, for all $\mathrm{V}$-recognizable languages $K, L \subseteq A^{*}$, the language $K L$ is also $\mathrm{V}$-recognizable. Similar notions may be considered for pseudovarieties of semigroups.

As shown in [7, Lemma 2.3] for pseudovarieties of semigroups, such a pseudovariety $\mathrm{V}$ containing all finite nilpotent semigroups is closed under concatenation if and only if the multiplication in $\bar{\Omega}_{A} \mathrm{~V}$ is an open mapping for each finite set $A$. As mentioned in Section 2, the requirement that $\mathrm{V}$ contain all finite nilpotent semigroups is made to ensure that the subsemigroup 
of $\bar{\Omega}_{A} \vee$ generated by the free generators is free and discrete. In the case of pseudovarieties of monoids, the corresponding sufficient condition is that $\mathrm{V}$ contain MN. Under such an assumption for a pseudovariety of monoids $\mathrm{V}$, the argument in the proof of [7, Lemma 2.3] also yields that $\mathrm{V}$ is closed under concatenation if and only if the multiplication in $\bar{\Omega}_{A} \mathrm{~V}$ is an open mapping for each finite set $A$.

Another relevant result from the same paper is [7, Lemma 4.8], whose proof shows that, if the multiplication in $\bar{\Omega}_{A} \mathrm{~V}$ is an open mapping, then $\bar{\Omega}_{A} \mathrm{~V}$ is equidivisible. In particular, since the pseudovariety $\mathrm{A}$ is closed under concatenation, the multiplication in each profinite aperiodic monoid $\bar{\Omega}_{A} \mathrm{~A}$ is an open mapping and $A$ is equidivisible.

For an $\omega$-term $s$ and a positive integer $k$, the $\omega$-term $s^{k}$ is defined recursively by $s^{1}=s$ and $s^{k+1}=s^{k} \cdot s$. The need for expliciting this definition is due to the fact that the multiplication of $\omega$-terms is not associative, as we prefer to keep track of the order in which the operations are performed. Additionally, we assume that, for all $\omega$-terms $s$ and $t$, both $s^{0} \cdot t$ and $t \cdot s^{0}$ denote $t$.

Decompositions of an $\omega$-term $t$ are pairs of $\omega$-terms, defined inductively with respect to the structure of $t$. If $t$ is either 1 or a letter, then its decompositions are $(1, t)$ and $(t, 1)$. If $t=t_{1} \cdot t_{2}$, then $t$ has decompositions of two symmetric forms:

- for every decomposition $\left(s_{1}, s_{2}\right)$ of $t_{1}$, the pair $\left(s_{1}, s_{2} \cdot t_{2}\right)$ is a decomposition of $t$;

- for every decomposition $\left(s_{1}, s_{2}\right)$ of $t_{2}$, the pair $\left(t_{1} \cdot s_{1}, s_{2}\right)$ is a decomposition of $t$.

If $t=s^{\omega}$, then the decompositions of $t$ are defined to be the pairs of the form $\left(s^{k} \cdot s_{1}, s_{2} \cdot s^{\ell}\right)$, where $\left(s_{1}, s_{2}\right)$ is a decomposition of $s$ and $k, \ell$ are non-negative integers or $\omega$, with at least one of them equal to $\omega$.

In order to illustrate the definition with a concrete example, let $a, b$ be a pair of letters. Decompositions of the term $a \cdot b$ are exactly $(1, a \cdot b),(a, 1 \cdot b)$, $(a \cdot 1, b)$ and $(a \cdot b, 1)$. Consequently, decompositions of the term $(a \cdot b)^{\omega}$ are

$$
\begin{array}{ll}
\left((a \cdot b)^{k} \cdot 1,(a \cdot b) \cdot(a \cdot b)^{\ell}\right), & \left((a \cdot b)^{k} \cdot a,(1 \cdot b) \cdot(a \cdot b)^{\ell}\right), \\
\left((a \cdot b)^{k} \cdot(a \cdot 1), b \cdot(a \cdot b)^{\ell}\right), & \left((a \cdot b)^{k} \cdot(a \cdot b), 1 \cdot(a \cdot b)^{\ell}\right),
\end{array}
$$

where $k$ and $\ell$ are non-negative integers or $\omega$, with at least one of them equal to $\omega$. In particular, the decompositions obtained for $\ell=0$ and $k=\omega$ are

$$
\begin{array}{ll}
\left((a \cdot b)^{\omega} \cdot 1, a \cdot b\right), & \left((a \cdot b)^{\omega} \cdot a, 1 \cdot b\right), \\
\left((a \cdot b)^{\omega} \cdot(a \cdot 1), b\right), & \left((a \cdot b)^{\omega} \cdot(a \cdot b), 1\right) .
\end{array}
$$

Note that $\left((a \cdot b)^{\omega}, 1\right),\left((a \cdot b)^{\omega} \cdot a, b\right)$ and $\left((a \cdot b)^{\omega},(a \cdot b)^{\omega}\right)$ fail to be decompositions of $(a \cdot b)^{\omega}$. However, as the next result shows, all factorizations of $\omega$-words may be obtained from decompositions of $\omega$-terms representing them. It appears to be intimately related with results of [10, but not quite to follow from them in any direct way. We thus include here a direct proof. The key ingredients are the facts that $\bar{\Omega}_{A} \mathrm{~A}$ is equidivisible and its multiplication is an open mapping. 
Theorem 5.1. Let $t$ be an $\omega$-term and $u, v \in \bar{\Omega}_{A} \mathrm{~A}$. Then, $t$ represents the product uv if and only if there exists a decomposition $\left(t_{1}, t_{2}\right)$ of $t$ such that $t_{1}$ represents $u$ and $t_{2}$ represents $v$.

Proof. That a decomposition of $t$ yields a factorization of the $\omega$-word in $\Omega_{A}^{\omega} \mathrm{A}$ represented by $t$ amounts to a straightforward induction on the construction of $t$ as an $\omega$-term. For the converse, we also proceed by induction on the construction of $t$ as an $\omega$-term. At the basis of the induction are the identity term 1 and the letters, for which the result is obvious: indeed, both 1 and the letters admit no nontrivial factorizations in $\bar{\Omega}_{A} \mathrm{~A}$ and this corresponds exactly to the decompositions allowed in these cases.

Suppose that $t=s_{1} \cdot s_{2}$ is obtained by multiplying two (shorter) terms and let $w_{i}$ be the $\omega$-word in $\Omega_{A}^{\omega} \mathrm{A}$ represented by $s_{i}(i=1,2)$. From the equality $w_{1} w_{2}=u v$, by equidivisibility of $\bar{\Omega}_{A} \mathrm{~A}$ we deduce that there is some pseudoword $z \in \bar{\Omega}_{A} \mathrm{~A}$ such that either $w_{1}=u z$ and $z w_{2}=v$, or $w_{1} z=u$ and $w_{2}=z v$. In the first case, applying the induction hypothesis to $s_{1}$, we obtain a decomposition $\left(s_{1,1}, s_{1,2}\right)$ of $s_{1}$ such that $s_{1,1}$ represents $u$ and $s_{1,2}$ represents $z$. Hence, the $\omega$-terms $t_{1}=s_{1,1}$ and $t_{2}=s_{1,2} \cdot s_{2}$ represent $u$ and $v$, respectively. The other case is similar.

Suppose next that $t=s^{\omega}$ where $s$ is a (shorter) $\omega$-term. In view of the preceding case, we may assume inductively that the result holds for each $\omega$ term $s^{n}$ with $n$ a positive integer. Let $w$ be the $\omega$-word in $\Omega_{A}^{\omega}$ A represented by $s$. Since the sequence $\left(w^{n}\right)_{n}$ converges to the product $u v$ and the multiplication in $\bar{\Omega}_{A} \mathrm{~A}$ is open, there is a strictly increasing sequence of indices $\left(n_{k}\right)_{k}$ such that there is a factorization $w^{n_{k}}=u_{k} v_{k}$ with $\lim u_{k}=u$ and $\lim v_{k}=v$ (cf. [7, Lemma 2.5]). By the induction hypothesis applied to $s^{n_{k}}$, for each $k$ there is a decomposition $\left(t_{k, 1}, t_{k, 2}\right)$ of $s^{n_{k}}$ such that $t_{k, 1}$ represents $u_{k}$ and $t_{k, 2}$ represents $v_{k}$. Moreover, by the definition of decomposition and a simple induction, for each $k$ there exists $\ell_{k}$, with $0 \leqslant \ell_{k} \leqslant n_{k}-1$, such that $t_{k, 1}=s^{\ell_{k}} \cdot t_{k, 1}^{\prime}$ and $t_{k, 2}=\left(\ldots\left(\left(t_{k, 2}^{\prime} \cdot s\right) \cdot s\right) \ldots\right) \cdot s$, where $\left(t_{k, 1}^{\prime}, t_{k, 2}^{\prime}\right)$ is a decomposition of $s$ and in the latter term the number of multiplications by $s$ is $n_{k}-\ell_{k}-1$. Let $w_{k, i}$ be the $\omega$-word in $\Omega_{A}^{\omega} \mathrm{A}$ represented by $t_{k, i}^{\prime}(i=1,2)$. Then the equalities $u_{k}=w^{\ell_{k}} \cdot w_{k, 1}$ and $v_{k}=w_{k, 2} \cdot w^{n_{k}-\ell_{k}-1}$ hold in $\Omega_{A}^{\omega} \mathrm{A}$, and the decomposition of $s$ yields the factorization $w=w_{k, 1} w_{k, 2}$. Up to taking a subsequence of $\left(n_{k}\right)_{k}$, we may assume that each of the sequences $\left(w_{k, i}\right)_{k}$ converges to some $w_{i}(i=1,2)$ and that each of the sequences $\left(\ell_{k}\right)_{k}$ and $\left(n_{k}-\ell_{k}-1\right)_{k}$ is either constant or strictly increasing. Let $\ell$ denote the common value of $\ell_{k}$ if the sequence $\left(\ell_{k}\right)_{k}$ is constant, and $\omega$ if the sequence is strictly increasing. Similarly, let $m$ denote either the common value of $n_{k}-\ell_{k}-1$ or $\omega$. Continuity of multiplication in $\bar{\Omega}_{A} \mathrm{~A}$ yields the equalities $u=w^{\ell} w_{1}, v=w_{2} w^{m}$, and $w=w_{1} w_{2}$. The latter equality and the induction hypothesis applied to the $\omega$-term $s$ provide a decomposition $\left(s_{1}, s_{2}\right)$ of $s$ such that $s_{i}$ represents $w_{i}(i=1,2)$. It remains to take $t_{1}=s^{\ell} \cdot s_{1}$ and $t_{2}=s_{2} \cdot s^{m}$ to obtain the required decomposition.

A subset $X$ of a semigroup $S$ is said to be factorial in $S$ if $s t \in X$ with $s, t \in S$ implies $s, t \in X$. For an implicit signature $\sigma$, a pseudovariety $\mathrm{V}$ is said to be $\sigma$-factorial if $\Omega_{A}^{\sigma} \bigvee$ is factorial in $\bar{\Omega}_{A} \bigvee$ for every finite set $A$. 
The following result is an immediate application of Theorem [5.1. It was first proved in [9] as a by-product of a language-theoretical proof of the correctness of McCammond's algorithm for solving the $\omega$-identity problem for A [17. An alternative proof and a generalization to all Burnside pseudovarieties $B_{n}=\llbracket x^{\omega+n}=x^{\omega} \rrbracket$ can be found in [10].

Corollary 5.2. The pseudovariety A is $\omega$-factorial.

Combining Corollary 5.2 with Corollary 3.2 , we obtain the following result.

Corollary 5.3. Let $\mathrm{V}$ be a polynomially closed pseudovariety of aperiodic ordered monoids and let $u, v \in \Omega_{A}^{\omega} \mathrm{A}$. If the inequality $u \leqslant v$ holds in $\mathrm{V}$ then, for every factorization $u=u_{1} \cdots u_{n}$, there is a factorization $v=v_{1} \cdots v_{n}$ into $\omega$-words such that each inequality $u_{i} \leqslant v_{i}$ holds in $\mathrm{V}(i=1, \ldots, n)$.

\section{Repetitions IN $\omega$-WORDS}

This section is devoted to establishing another reflection of the intuitively expected phenomenon that there are not many ways to factorize an $\omega$-word. A precise formulation of how such factorizations are obtained is already given by Theorem 5.1. For application in the next section, we also need the following repetition result for iterated factorizations.

Proposition 6.1. Suppose, for each $i \geqslant 0, v_{i}, x_{i}$ are $\omega$-words in $\Omega_{A}^{\omega} \mathrm{A}$ such that $v_{i}=x_{i} v_{i+1}$. Then there exist indices $i$ and $j$ with $i \leqslant j$ such that $v_{i}=\left(x_{i} \cdots x_{j}\right)^{\omega} v_{j+1}$.

As an example, take $v_{0}=\left(a^{2} b^{\omega}\right)^{\omega} a b^{\omega}$ and consider the factorizations $v_{i}=x_{i} v_{i+1}$ given by the $\omega$-words $v_{i}=b^{i} v_{0}$ and $x_{i}=b^{i}\left(a^{2} b^{\omega}\right)^{\omega}(i \geqslant 0)$. Note that there are infinitely many ways to factorize $v_{0}$. However, Proposition 6.1 says that some repetition is always possible. In this concrete example, this is quite easy, because one can take $i=j$ an arbitrary index. In the general case we need to be more careful to choose appropriate indices and we need to overcome certain technical obstacles.

In order to prove this key proposition, we are going to study, instead of the factorizations $v_{i}=x_{i} v_{i+1}$, the corresponding syntactic decompositions of an $\omega$-term representing $v_{0}$, with the aim of finding certain repetitions, which would allow to repeat several consecutive $\omega$-terms in the decomposition without changing the resulting $\omega$-word $v_{i}=x_{i} \cdots x_{j} v_{j+1}$.

Lemma 6.2. Let $v \in \Omega_{A}^{\omega} \mathrm{A}$ be an $\omega$-word, and for each $i \geqslant 1$ let $v=v_{i} \tilde{v}_{i}$ be a factorization in $\Omega_{A}^{\omega} \mathrm{A}$. Then there exist indices $i$ and $j$ with $i<j$ such that $v=v_{j} \tilde{v}_{i}$.

Proof. Let $t$ be an $\omega$-term representing $v$. We proceed by induction on the construction of $t$. If $t$ is a letter from $A$, then the only factorizations of $v$ are $1 \cdot v$ and $v \cdot 1$ by Theorem [5.1, and the claim is obvious.

In case $t=t_{1} \cdot t_{2}$, denote by $u_{1}$ and $u_{2}$ the $\omega$-words represented by $t_{1}$ and $t_{2}$, respectively. By equidivisibility, for each $i$ there exists $y_{i} \in \Omega_{A}^{\omega} \mathrm{A}$ such that either $u_{1}=v_{i} y_{i}$ and $y_{i} u_{2}=\tilde{v}_{i}$, or $u_{1} y_{i}=v_{i}$ and $u_{2}=y_{i} \tilde{v}_{i}$. By symmetry, we may assume that the former case occurs infinitely often. Applying the induction hypothesis to the factorizations of $u_{1}$, we obtain indices $i$ and $j$ 
with $i<j$ such that $u_{1}=v_{j} y_{i}$. It follows that $v=u_{1} u_{2}=v_{j} y_{i} u_{2}=v_{j} \tilde{v}_{i}$, as required.

Finally, consider the case $t=s^{\omega}$. According to Theorem 5.1, for each $i$ there exists a decomposition $\left(s_{i, 1}, s_{i, 2}\right)$ of the $\omega$-term $s$ and exponents $k_{i}, \ell_{i}$, that are either non-negative integers or $\omega$, such that $v_{i}$ is represented by the $\omega$-term $s^{k_{i}} \cdot s_{i, 1}$ and $\tilde{v}_{i}$ is represented by the $\omega$-term $s_{i, 2} \cdot s^{\ell_{i}}$. Since for each $i$ at least one of $k_{i}$ and $\ell_{i}$ is equal to $\omega$, without loss of generality we may assume that $\ell_{i}=\omega$ for all $i$. Consider the $\omega$-words $w_{i}$ and $\tilde{w}_{i}$ represented by the $\omega$-terms $s_{i, 1}$ and $s_{i, 2}$, respectively. Then, all products $w_{i} \tilde{w}_{i}$ are equal to the $\omega$-word $u$ represented by $s$. Using the induction hypothesis, we obtain indices $i$ and $j$ with $i<j$ such that $u=w_{j} \tilde{w}_{i}$. Then $v=u^{\omega}=u^{k_{j}} w_{j} \tilde{w}_{i} u^{\omega}=v_{j} \tilde{v}_{i}$, which concludes the proof.

For the proof of Proposition 6.1, it is convenient to introduce a measure of depth of $\omega$-terms. With this aim, let $t$ be an arbitrary $\omega$-term. In terms of the tree of $t$, we consider two parameters:

- the maximum number $\mu_{\omega}(t)$ of occurrences of the $\omega$-power in a branch of the tree of $t$;

- for each branch of the tree of $t$ with $\mu_{\omega}(t)$ occurrences of the $\omega$-power, let $n$ be the number of right descendants above the top occurrence of the $\omega$-power in the branch; the number $\mu_{\ell}(t)$ is the maximum of all such $n$ (if $\mu_{\omega}(t)=0$, then $\mu_{\ell}(t)$ is equal to the maximum number of right descendants in a branch).

Let $\mu(t)$ stand for the pair $\left(\mu_{\omega}(t), \mu_{\ell}(t)\right)$. For example, for the term $t$ depicted in Figure 1, we have $\mu(t)=(2,0)$, while if the term $s$ is obtained by replacing in $t$ the rightmost leaf $b$ by the term $b^{\omega}$, then $\mu(s)=(2,1)$.

Using the lexicographic ordering $\leqslant$ of pairs of non-negative integers, the definition of $\mu$ can be rephrased inductively with respect to the structure of terms as follows: $\mu(1)=\mu(a)=(0,0)$ for all $a \in A ; \mu\left(s^{\omega}\right)=\left(\mu_{\omega}(s)+1,0\right)$; $\mu\left(t_{1} \cdot s^{\omega}\right)=\max \left\{\mu\left(t_{1}\right), \mu\left(s^{\omega}\right)\right\} ; \mu\left(t_{1} \cdot t_{2}\right)=\max \left\{\mu\left(t_{1}\right), \mu\left(t_{2}\right)+(0,1)\right\}$ if $t_{2}$ is not of the form $s^{\omega}$, where the addition of pairs is taken component-wise.

Lemma 6.3. If $t$ is an $\omega$-term and $\left(\bar{t}, t^{\prime}\right)$ is a decomposition of $t$, then $\mu(t) \geqslant$ $\mu\left(t^{\prime}\right)$ and the equality can only occur in the following four cases:

(1) $t$ is 1 or a letter;

(2) $t=s_{1} \cdot s_{2}$ and $\left(\bar{t}, t^{\prime}\right)=\left(\bar{s}, s^{\prime} \cdot s_{2}\right)$ for some decomposition $\left(\bar{s}, s^{\prime}\right)$ of $s_{1}$;

(3) $t=s^{\omega}$ and $\left(\bar{t}, t^{\prime}\right)=\left(s^{k} \cdot \bar{s}, s^{\prime} \cdot s^{\omega}\right)$ for some decomposition $\left(\bar{s}, s^{\prime}\right)$ of $s$ and $k \in\{0,1,2, \ldots\} \cup\{\omega\}$;

(4) $t=s_{1} \cdot s^{\omega}$ and $\left(\bar{t}, t^{\prime}\right)=\left(s_{1} \cdot\left(s^{k} \cdot \bar{s}\right), s^{\prime} \cdot s^{\omega}\right)$ for some decomposition $\left(\bar{s}, s^{\prime}\right)$ of $s$ and $k \in\{0,1,2, \ldots\} \cup\{\omega\}$.

Proof. We proceed by induction on the construction of the term $t$. The claim is obvious for $t$ equal to 1 or a letter, which means that we fall in Case (11).

Suppose that $t=s_{1} \cdot s_{2}$ and $\left(\bar{t}, t^{\prime}\right)=\left(\bar{s}, s^{\prime} \cdot s_{2}\right)$, with $\left(\bar{s}, s^{\prime}\right)$ a decomposition of $s_{1}$. Since these assumptions fall in Case (2), it is enough to prove the inequality $\mu(t) \geqslant \mu\left(t^{\prime}\right)$. We distinguish two cases. For $s_{2}$ of the form $s^{\omega}$ we get

$$
\mu(t)=\max \left\{\mu\left(s_{1}\right), \mu\left(s^{\omega}\right)\right\} \geqslant \max \left\{\mu\left(s^{\prime}\right), \mu\left(s^{\omega}\right)\right\}=\mu\left(s^{\prime} \cdot s_{2}\right),
$$


using the induction hypothesis on the $\omega$-term $s_{1}$. Similarly, if $s_{2}$ is not of the form $s^{\omega}$, we obtain

$$
\mu(t)=\max \left\{\mu\left(s_{1}\right), \mu\left(s_{2}\right)+(0,1)\right\} \geqslant \max \left\{\mu\left(s^{\prime}\right), \mu\left(s_{2}\right)+(0,1)\right\}=\mu\left(s^{\prime} \cdot s_{2}\right) .
$$

If $t=s_{1} \cdot s_{2}$ and $\left(\bar{t}, t^{\prime}\right)=\left(s_{1} \cdot \bar{s}, s^{\prime}\right)$, with $\left(\bar{s}, s^{\prime}\right)$ a decomposition of $s_{2}$, then we also distinguish two cases. For $s_{2}$ of the form $s^{\omega}$ we get

$$
\mu(t)=\max \left\{\mu\left(s_{1}\right), \mu\left(s^{\omega}\right)\right\} \geqslant \mu\left(s^{\omega}\right)=\mu\left(s_{2}\right) \geqslant \mu\left(s^{\prime}\right),
$$

using the induction hypothesis on the $\omega$-term $s_{2}$; in fact, the induction hypothesis yields that the last inequality is strict unless $\left(\bar{s}, s^{\prime}\right)$ is of the form $\left(s^{k} \cdot \tilde{s}, s^{\prime \prime} \cdot s^{\omega}\right)$ for some decomposition $\left(\tilde{s}, s^{\prime \prime}\right)$ of $s$ and $k \in\{0,1,2, \ldots\} \cup\{\omega\}$, which shows that the equality $\mu(t)=\mu\left(s^{\prime}\right)$ only holds in Case (4). In the second case, assuming that $s_{2}$ is not of the form $s^{\omega}$, we obtain strict inequality:

$$
\mu(t)=\max \left\{\mu\left(s_{1}\right), \mu\left(s_{2}\right)+(0,1)\right\}>\mu\left(s_{2}\right) \geqslant \mu\left(s^{\prime}\right) .
$$

If $t=s^{\omega}$ and the decomposition $\left(\bar{t}, t^{\prime}\right)$ of $t$ has $t^{\prime}=s^{\prime} \cdot s^{\omega}$, with $\left(\bar{s}, s^{\prime}\right)$ a decomposition of $s$, meaning that Case (3) holds, then

$$
\mu\left(s^{\prime} \cdot s^{\omega}\right)=\max \left\{\mu\left(s^{\prime}\right), \mu\left(s^{\omega}\right)\right\}=\mu\left(s^{\omega}\right),
$$

because $\mu\left(s^{\prime}\right) \leqslant \mu(s)<\mu\left(s^{\omega}\right)$ by the induction hypothesis. Finally, if $t=s^{\omega}$ and the decomposition $\left(\bar{t}, t^{\prime}\right)$ of $t$ has $t^{\prime}=s^{\prime} \cdot s^{k}$, with $\left(\bar{s}, s^{\prime}\right)$ a decomposition of $s$ and $k$ a non-negative integer, then

$$
\mu\left(s^{\prime} \cdot s^{k}\right) \leqslant \max \left\{\mu\left(s^{\prime}\right),\left(\mu_{\omega}(s), \mu_{\ell}(s)+2\right)\right\}<\mu\left(s^{\omega}\right),
$$

because $\mu\left(s^{\prime}\right) \leqslant \mu(s)<\mu\left(s^{\omega}\right)$ by the induction hypothesis, while a simple induction taking into account that $s^{k+1}=s^{k} \cdot s$ shows that the equality $\mu\left(s^{k}\right)=\mu(s)+(0,1)$ holds whenever $k>1$ in case $s$ is not of the form $s=r^{\omega}$, and $\mu\left(s^{k}\right)=\mu(s)$ otherwise.

We may now proceed with the proof of the main result of this section.

Proof of Proposition 6.1. Let $t_{0}$ be an $\omega$-term representing $v_{0}$. Using Theorem 5.1, choose successively for each $i$ some decomposition $\left(s_{i}, t_{i+1}\right)$ of the $\omega$-term $t_{i}$ such that $s_{i}$ represents $x_{i}$ and $t_{i+1}$ represents $v_{i+1}$.

The proof of the proposition proceeds by induction with respect to $\mu\left(t_{0}\right)$, and for $\omega$-terms $t_{0}$ with the same value of $\mu\left(t_{0}\right)$ by induction with respect to the size of the term. By Lemma 6.3, we know that $\mu\left(t_{i+1}\right) \leqslant \mu\left(t_{i}\right)$ for every $i \geqslant 0$. If $\mu\left(t_{i}\right)<\mu\left(t_{0}\right)$ for some $i \geqslant 1$, then the statement follows directly from the induction assumption applied to $t_{i}$. Thus, assume that $\mu\left(t_{i}\right)=\mu\left(t_{0}\right)$ for all $i$, which implies that all decompositions $\left(s_{i}, t_{i+1}\right)$ are of one of the four forms described in Lemma 6.3.

If some term $t_{i}$ is 1 or a letter, then the same is true for all terms $t_{i}$, and all terms $s_{i}$, except for at most one, are equal to 1 ; then the statement is obviously true. It remains to deal with the situation when every decomposition $\left(s_{i}, t_{i+1}\right)$ is of one of the forms (2) to (4), which is assumed for the remainder of the proof. Let us first formulate a useful observation.

Claim. Assume that $i$ is such that the decomposition $\left(s_{i}, t_{i+1}\right)$ of $t_{i}$ is of the form (3) with $t_{i}=s^{\omega}$ or (4) with $t_{i}=\bar{t}_{i} \cdot s^{\omega}$ for some $\omega$-terms $s$ and $\bar{t}_{i}$. Then, for every $j>i$, the decomposition $\left(s_{j}, t_{j+1}\right)$ is of the form (21) or (44), 
and there exist $\omega$-terms $\bar{t}_{j}$ for $j>i$ such that $t_{j}=\bar{t}_{j} \cdot s^{\omega}$. Moreover, the inequality $\mu\left(\bar{t}_{i+1}\right)<\mu\left(t_{i+1}\right)$ holds.

Proof of Claim. We prove the statement that $t_{j}=\bar{t}_{j} \cdot s^{\omega}$ for some $\omega$-terms $\bar{t}_{j}$ by induction with respect to $j>i$. Note that since $\mu\left(t_{j+1}\right)=\mu\left(t_{j}\right)$, this form of $t_{j}$ ensures that, for $j>i$, the decomposition $\left(s_{j}, t_{j+1}\right)$ of $t_{j}$ is of the form (2) or (4).

By the assumption that the decomposition $\left(s_{i}, t_{i+1}\right)$ of $t_{i}$ is of the form (3) or (4), we know that the $\omega$-term $t_{i+1}$ has the form $\bar{t}_{i+1} \cdot s^{\omega}$ where $\bar{t}_{i+1}=s^{\prime}$ in the notation of Lemma 6.3. Thus, we have proved the base of the induction $j=i+1$. Moreover, in both cases the term $s^{\prime}$ is the second component of a decomposition $\left(\bar{s}, s^{\prime}\right)$ of $s$ and, therefore, $\mu\left(\bar{t}_{i+1}\right)=\mu\left(s^{\prime}\right) \leqslant \mu(s)$ by Lemma 6.3. Hence we get the last statement of the claim, because $\mu(s)<$ $\mu\left(s^{\omega}\right) \leqslant \mu\left(t_{i+1}\right)$.

To prove the induction step, we first assume that the decomposition $\left(s_{j}, t_{j+1}\right)$ of $t_{j}=\bar{t}_{j} \cdot s^{\omega}$, with $j>i$, is of the form (2). Then, we have $t_{j+1}=t^{\prime}=s^{\prime} \cdot s^{\omega}$ in the notation of case (2) of Lemma 6.3, where $s^{\omega}=s_{2}$. Hence, we may take $\bar{t}_{j+1}=s^{\prime}$ and we are done in this case. We may proceed in the same way when we assume that the decomposition $\left(s_{j}, t_{j+1}\right)$ of $t_{j}=\bar{t}_{j} \cdot s^{\omega}$, with $j>i$, is of the form (44).

We distinguish two cases for which the proof proceeds in different ways. First, assume that there exists an index $k$ such that for all $j \geqslant k$ the decomposition $\left(s_{j}, t_{j+1}\right)$ of $t_{j}$ is of the form (2), that is, there exist $\omega$-terms $t^{\prime}$ and $\bar{t}_{j}$ for $j \geqslant k$ such that $t_{j}=\bar{t}_{j} \cdot t^{\prime}$ and $\left(s_{j}, \bar{t}_{j+1}\right)$ is a decomposition of $\bar{t}_{j}$. Choose $k$ to be the least of such indices. Denote by $\bar{v}_{j}$ the $\omega$-word represented by $\bar{t}_{j}$. Our goal is to apply the induction hypothesis to the sequence of factorizations $\bar{v}_{j}=x_{j} \bar{v}_{j+1}$ for $j \geqslant k$. This would be possible, once we verify either that $\mu\left(\bar{t}_{k}\right)<\mu\left(t_{0}\right)$ or that $\mu\left(\bar{t}_{k}\right)=\mu\left(t_{0}\right)$ and the size of $\bar{t}_{k}$ is smaller than that of $t_{0}$. At least one of these conditions is certainly true if $k=0$, since then $\bar{t}_{k}$ is a proper subterm of $t_{0}$. If $k \geqslant 1$, then the minimality of choice of $k$ ensures that the decomposition $\left(s_{k-1}, t_{k}\right)$ of $t_{k-1}$ is of the form (3) or (4). Hence, we may apply the Claim with $i=k-1$ to deduce that $\mu\left(\bar{t}_{k}\right)<\mu\left(t_{k}\right)$. Since we have also assumed that $\mu\left(t_{k}\right)=\mu\left(t_{0}\right)$, the induction hypothesis now provides us with indices $i$ and $j$, with $i<j$, such that $\bar{v}_{i}=\left(x_{i} \cdots x_{j}\right)^{\omega} \bar{v}_{j+1}$. Multiplication by the $\omega$-word $w$ represented by the $\omega$-term $t^{\prime}$ turns this equality into the required repetition $v_{i}=\bar{v}_{i} w=\left(x_{i} \cdots x_{j}\right)^{\omega} \bar{v}_{j+1} w=\left(x_{i} \cdots x_{j}\right)^{\omega} v_{j+1}$.

Second, assume that such an index $k$ does not exist, so that, according to the claim, for infinitely many indices $i$ the decomposition $\left(s_{i}, t_{i+1}\right)$ is of the form (44). Let $i_{1}<i_{2}<\cdots$ be all such indices. Then for every $m \geqslant 1$, all decompositions with indices between $i_{m}$ and $i_{m+1}$ are of the form (2). For all $i \geqslant i_{1}$, the term $t_{i}$ can be written as $t_{i}=\bar{t}_{i} \cdot s^{\omega}$, for a fixed $\omega$-term $s$ and certain $\omega$-terms $\bar{t}_{i}$. If $i$ is not one of the indices $i_{m}$, then $\left(s_{i}, \bar{t}_{i+1}\right)$ is a decomposition of $\bar{t}_{i}$. On the other hand, for all indices $i_{m}$ we have $s_{i_{m}}=\bar{t}_{i_{m}} \cdot\left(s^{k_{m}} \cdot \bar{s}_{m}\right)$, with $\left(\bar{s}_{m}, \bar{t}_{i_{m}+1}\right)$ a decomposition of $s$ and $k_{m} \in\{0,1,2, \ldots\} \cup\{\omega\}$. Denoting $\omega$-words in $\Omega_{A}^{\omega} \mathrm{A}$ represented by $\omega$-terms $\bar{t}_{i}, s$ and $\bar{s}_{m}$ by $\bar{v}_{i}, w$ and $\bar{w}_{m}$, respectively, the above relations between these $\omega$-terms translate into the following equalities of $\omega$-words: $v_{i}=\bar{v}_{i} w^{\omega}$ for all $i \geqslant i_{1}, \bar{v}_{i}=x_{i} \bar{v}_{i+1}$ for indices $i$ other than $i_{m}, x_{i_{m}}=\bar{v}_{i_{m}} w^{k_{m}} \bar{w}_{m}$, and 
$w=\bar{w}_{m} \bar{v}_{i_{m}+1}$. By Lemma 6.2. there exist indices $\ell, n \geqslant 1$, with $\ell<n$, such that $w=\bar{w}_{n} \bar{v}_{i_{\ell}+1}$. We are going to verify that $i_{\ell}+1$ and $i_{n}$ are the required indices $i$ and $j$. First we calculate the product of $\omega$-terms $x_{i}$ between two consecutive indices $i_{m}$ as

$$
x_{i_{m}+1} \cdots x_{i_{m+1}}=x_{i_{m}+1} \cdots x_{i_{m+1}-1} \bar{v}_{i_{m+1}} w^{k_{m+1}} \bar{w}_{m+1}=\bar{v}_{i_{m}+1} w^{k_{m+1}} \bar{w}_{m+1} \text {, }
$$

using successively the equalities $\bar{v}_{i}=x_{i} \bar{v}_{i+1}$ for $i=i_{m+1}-1, \ldots, i_{m}+1$. Then, we obtain

$$
x_{i_{\ell}+1} \cdots x_{i_{n}}=\bar{v}_{i_{\ell}+1} w^{k_{\ell+1}+\cdots+k_{n}+n-\ell-1} \bar{w}_{n},
$$

using all equalities $w=\bar{w}_{m} \bar{v}_{i_{m}+1}$ for $m=\ell+1, \ldots, n-1$. Denoting the number $k_{\ell+1}+\cdots+k_{n}+n-\ell-1$ by $q$, we finally calculate

$$
\begin{aligned}
\left(x_{i_{\ell}+1} \cdots x_{i_{n}}\right)^{\omega} v_{i_{n}+1} & =\left(\bar{v}_{i_{\ell}+1} w^{q} \bar{w}_{n}\right)^{\omega} \bar{v}_{i_{n}+1} w^{\omega} \\
& =\bar{v}_{i_{\ell}+1}\left(w^{q} \bar{w}_{n} \bar{v}_{i_{\ell}+1}\right)^{\omega} w^{q} \bar{w}_{n} \bar{v}_{i_{n}+1} w^{\omega} \\
& =\bar{v}_{i_{\ell}+1} w^{\omega}=v_{i_{\ell}+1},
\end{aligned}
$$

using the equalities $\bar{w}_{n} \bar{v}_{i_{\ell}+1}=\bar{w}_{n} \bar{v}_{i_{n}+1}=w$.

\section{Syntactic Proofs of $\omega$-INeQUalities}

The aim of this section is to show that the positive cases of the $\omega$-inequality problem for an aperiodic pseudovariety of ordered monoids of the form $\mathrm{W}=$ Pol BV may be derived from the positive cases of the $\omega$-inequality problem for V. Since we deal only with aperiodic monoids, it is convenient to consider only $\omega$-inequality problems over $\mathrm{A}$. By Proposition 4.2 , the general $\omega$-inequality problem reduces to this one since the $\omega$-equality problem for $A$ is decidable as was shown by McCammond [17. For this reason, all $\omega$-inequalities considered in this section are taken over $A$.

In order to enumerate the positive cases of the $\omega$-inequality problem for $\mathrm{W}$, we show that, under suitable assumptions on $\mathrm{V}$, if an inequality $u \leqslant v$ of $\omega$-words is valid in $\mathrm{W}$, then there is a finite syntactic proof of this fact from a convenient set of hypotheses, or axioms. This is thus a sort of completeness result for the choice of the set of axioms. Provided the axioms can be enumerated, since formal proofs can then be enumerated, so can be the provable facts.

To fulfill the above program, we need to make precise what kind of formal proofs we will be considering. By a formal proof of an $\omega$-inequality $u \leqslant v$ from a given set $\Gamma$ of hypotheses, we mean a finite sequence $u_{i} \leqslant v_{i}(i=$ $1, \ldots, n)$ of $\omega$-inequalities such that each member of the sequence satisfies one of the following conditions:

- $u_{i} \leqslant v_{i}$ is a member of $\Gamma$;

- $u_{i} \leqslant v_{i}$ is of the form $u_{j} u_{k} \leqslant v_{j} v_{k}$ with $j, k<i$;

- $u_{i} \leqslant v_{i}$ is of the form $u_{j}^{\omega} \leqslant v_{j}^{\omega}$ with $j<i$;

- there are $j, k<i$ such that $u_{i}=u_{j}, v_{j}=u_{k}$, and $v_{k}=v_{i}$.

If there is such a formal proof, then we say that $u \leqslant v$ is provable from $\Gamma$ and we write $\Gamma \vdash u \leqslant v$. Note that we do not include in our proof rules the possibility of making substitutions of variables by $\omega$-words. We will not need them because we will consider sets of hypotheses that are closed under such substitutions. Similarly, we do not need to take into account the possibility 
of multiplying both sides of an inequality on the left and on the right by the same $\omega$-words because we will consider sets of hypotheses containing all trivial $\omega$-inequalities.

Proposition 7.1. Let $\mathrm{V}$ be a polynomially closed pseudovariety of aperiodic ordered monoids and let $\Gamma$ be the set of all trivial $\omega$-inequalities together with all inequalities of the form $u^{\omega} \leqslant u^{\omega} v u^{\omega}$, with $u, v \in \Omega_{A}^{\omega} \mathrm{A}$, such that the $\omega$-inequality $v \leqslant u$ is valid in $\mathrm{V}$. If an $\omega$-inequality $u \leqslant v$ is valid in $\mathrm{W}=\mathrm{PolB} \vee$, then $\Gamma \vdash u \leqslant v$.

Proof. Let $t$ be an $\omega$-term representing $u$. We proceed by induction on the construction of $t$. If $t$ is a letter or 1 , then $u=v$, and so $u=v$ belongs to $\Gamma$.

In case $t=t_{1} \cdot t_{2}$, we obtain the corresponding factorization $u=u_{1} u_{2}$, where $t_{i}$ represents $u_{i}(i=1,2)$. By Corollary 5.3, there is a factorization $v=v_{1} v_{2}$ in $\Omega_{A}^{\omega} \mathrm{A}$ such that the inequality $u_{i} \leqslant v_{i}$ is valid in $\mathrm{W}$. The induction hypothesis yields $\Gamma \vdash u_{i} \leqslant v_{i}(i=1,2)$ and so also $\Gamma \vdash u \leqslant v$.

Suppose next that $t=s^{\omega}$. Let $v_{0}=v$ and let $w \in \Omega_{A}^{\omega} \mathrm{A}$ be the $\omega$-word represented by $s$. Inductively, we apply Corollary 5.3 to the inequality $u \leqslant v_{i}$ $(i \geqslant 0)$ and the factorization $u=w u$, to obtain a factorization $v_{i}=x_{i} v_{i+1}$ in $\Omega_{A}^{\omega} \mathrm{A}$ such that the inequalities $w \leqslant x_{i}$ and $u \leqslant v_{i+1}$ hold in $\mathrm{W}$. By Proposition 6.1, there exist $i, j$ such that $0 \leqslant i \leqslant j$ and

$$
v=x_{0} \cdots x_{i-1}\left(x_{i} \cdots x_{j}\right)^{\omega} v_{j+1} .
$$

Similarly, by the left-right dual of Proposition 6.1, there exists a factorization $v_{j+1}=v^{\prime}\left(y_{n} \cdots y_{m}\right)^{\omega} y_{m-1} \cdots y_{0}$ in $\Omega_{A}^{\omega} \mathrm{A}$ such that each of the inequalities $u \leqslant v^{\prime}$ and $w \leqslant y_{p}(p=0, \ldots, n)$ holds in $\mathbf{W}$. Combining with (1), we obtain the factorization

$$
v=x_{0} \cdots x_{i-1}\left(x_{i} \cdots x_{j}\right)^{\omega} v^{\prime}\left(y_{n} \cdots y_{m}\right)^{\omega} y_{m-1} \cdots y_{0} .
$$

Since the pseudovariety of monoids generated by $\mathrm{V}$ is contained in $\mathrm{W}$, it must satisfy the equality $u=v^{\prime}$, whence $\mathrm{V}$ satisfies the inequality $v^{\prime} \leqslant u$. By the choice of $\Gamma$, it follows that the inequality $u^{\omega} \leqslant u^{\omega} v^{\prime} u^{\omega}$ belongs to $\Gamma$. The preceding inequality may be written as

$$
u \leqslant\left(w^{j-i+1}\right)^{\omega} v^{\prime}\left(w^{m-n+1}\right)^{\omega} .
$$

Since the inequality (3) belongs to $\Gamma$ and $u=w u=u w$ by aperiodicity, we deduce that

$$
\Gamma \vdash u \leqslant w^{i}\left(w^{j-i+1}\right)^{\omega} v^{\prime}\left(w^{m-n+1}\right)^{\omega} w^{m} .
$$

On the other hand, the term induction hypothesis yields the relations

$$
\Gamma \vdash w \leqslant x_{p} \text { and } \Gamma \vdash w \leqslant y_{q} \quad(p=0, \ldots, j ; q=0, \ldots, n) .
$$

Combining (2), (44), and (5), we conclude that $\Gamma \vdash u \leqslant v$, which completes the induction step and the proof.

\section{MAIN RESUlts}

By an ordered $\omega$-monoid we mean an ordered monoid with a unary operation of $\omega$-power such that $s \leqslant t$ implies $s^{\omega} \leqslant t^{\omega}$. A variety of ordered $\omega$-monoids is a class of such structures that is closed under taking homomorphic images, ordered $\omega$-submonoids, and arbitrary direct products. It is 
well known that varieties of ordered $\omega$-monoids are defined by $\omega$-inequalities [12, 16.

For a pseudovariety $\mathrm{V}$ of ordered monoids, we denote by $\mathrm{V}^{\omega}$ the variety of ordered $\omega$-monoids generated by $\mathrm{V}$. Taking into account the basis of $\omega$ identities of $A^{\omega}$ obtained by McCammond [17], Proposition 7.1 yields the following basis result.

Theorem 8.1. Let $\mathrm{W}$ be a pseudovariety of aperiodic monoids and let $\mathrm{V}=$ $\mathrm{Pol} \mathrm{W}$. Then the variety $(\mathrm{PolBV})^{\omega}$ of ordered $\omega$-monoids is defined by the following inequalities:

(1) $x(y z)=(x y) z, x(y x)^{\omega}=(x y)^{\omega} x$;

(2) $\left(x^{\omega}\right)^{\omega}=\left(x^{r}\right)^{\omega}=x x^{\omega}=x^{\omega} x=x^{\omega}$ for every $r \geqslant 2$;

(3) $u^{\omega} \leqslant u^{\omega} v u^{\omega}$ whenever the inequality $v \leqslant u$ is valid in $\mathrm{V}$.

Proof. The equalities in (11) and (2) hold in the variety $A^{\omega}$, whence also in the subvariety $(\mathrm{Pol} \mathrm{B} \mathrm{V})^{\omega}$. The inequalities in (3) hold in $(\mathrm{Pol} \mathrm{BV})^{\omega}$ by Proposition 2.2. That every inequality valid in $(\mathrm{Pol} \mathrm{B} \mathrm{V})^{\omega}$ is a consequence of the inequalities (11)-(3) follows from Proposition [7.1.

The following is the announced recursive enumerability of the $\omega$-identity problem for suitable pseudovarieties of ordered monoids. It is formulated as a transfer result of that property along the Boolean-polynomial closure.

Theorem 8.2. Let $\mathrm{W}$ be a pseudovariety of aperiodic monoids and let $\mathrm{V}=$ $\mathrm{Pol} \mathrm{W}$. If the $\omega$-inequality problem for $\mathrm{V}$ is recursively enumerable, then so is it for Pol B V.

Proof. Consider the set $\Gamma$ defined in the statement of Proposition 7.1 By Proposition 7.1, each $\omega$-inequality over $A$ valid in $\mathrm{W}=$ Pol B V is provable from $\Gamma$. Since the converse follows from Proposition 2.2, we conclude that the $\omega$-inequalities over $\mathrm{A}$ that are valid in $\mathrm{W}$ are precisely those that are provable from $\Gamma$. As $\Gamma$ is recursively enumerable by hypothesis, the inequalities that are provable from $\Gamma$ constitute a recursively enumerable set. Hence the $\omega$ inequality problem for $\mathrm{W}$ over $\mathrm{A}$ is recursively enumerable, and therefore the $\omega$-inequality problem for $\mathrm{W}$ is also recursively enumerable by Proposition 4.2 .

Note that, from the definitions, it follows immediately that, if $\mathrm{V}$ is a recursively enumerable pseudovariety of ordered monoids, then so is Pol B V. Combining Theorem 8.2 with Proposition 4.1, we obtain the following main theorem of this paper.

Theorem 8.3. Let $\mathrm{W}$ be a recursively enumerable pseudovariety of aperiodic monoids and let $\mathrm{V}=\mathrm{Pol} \mathrm{W}$. If the $\omega$-inequality problem for $\mathrm{V}$ is decidable, then so is it for Pol B V.

In particular, this leads to the following application.

Corollary 8.4. Let $\mathrm{V}$ be a recursively enumerable pseudovariety of aperiodic monoids such that the $\omega$-inequality problem is decidable for Pol V. Then, the $\omega$-inequality problem is decidable for every level and half level of the concatenation hierarchy starting from $\mathrm{V}$. 
To obtain our main application, we need to be able to start the induction process with a pseudovariety of the form PolW for which the $\omega$-inequality problem is decidable. For the Straubing-Thérien hierarchy, we should start with W as the trivial pseudovariety, for which Pol W is the pseudovariety of ordered monoids defined by the inequality $1 \leqslant x$ [24, Proposition 8.4], and sometimes and henceforth denoted $\mathrm{J}^{+}$. The join with its dual, defined by the inequality $1 \geqslant x$, is the pseudovariety $\mathrm{J}$ of all finite $\mathcal{J}$-trivial monoids. The $\omega$-equality problem for $\mathrm{J}$ has been solved by the first author [1]. The solution consists in writing $\omega$-words in a canonical form, namely products of letters and $\omega$-powers of words that are products of distinct letters in increasing order (assuming a total order on the alphabet), in such a way that any factor adjacent to an $\omega$-power $u^{\omega}$ has at least one letter that does not appear in $u$.

Given a word $w=a_{1} \cdots a_{n} \in A^{*}$, with $a_{i} \in A$, we denote by $w \uparrow$ the language $A^{*} a_{1} A^{*} \cdots a_{n} A^{*}$, consisting of all words that admit $w$ as a subword. We also say that $w$ is a subword of a pseudoword $u \in \bar{\Omega}_{A} \mathrm{M}$ if $u \in \overline{w \uparrow}$. If $u \in \Omega_{A}^{\omega} \mathrm{M}$ is an $\omega$-word in canonical form over $\mathrm{J}$ then $w$ is a subword of $u$ if and only if it is a subword of $u^{(k)}$ for some positive integer $k$, where $u^{(k)}$ is obtained from $u$ by replacing each exponent $\omega$ by $k$ [2, Lemma 8.2.3], or equivalently, if $w$ is a subword of some word from the language $u^{(*)}$ defined by the regular expression obtained from $u$ by replacing each exponent $\omega$ by $*$.

Proposition 8.5. The $\omega$-inequality problem for $\mathrm{J}^{+}$is decidable.

Proof. Let $u \leqslant v$ be an $\omega$-inequality. Since the transformation of an $\omega$-word to its canonical form over $\mathrm{J}$ is effective, without loss of generality we may assume that $u$ and $v$ are in canonical form.

Since every $\mathrm{J}^{+}$-recognizable language is a finite union of languages of the form $w \uparrow$, we conclude from Proposition 2.4 that $\mathrm{J}^{+}$satisfies the inequality $u \leqslant v$ if and only if every subword of $u$ is also a subword of $v$. In view of the observation preceding the statement of this proposition, the latter condition can be decided by checking whether the regular language consisting of all subwords of $u^{(*)}$ is a subset of the regular language of subwords of $v^{(*)}$.

Finally, here is the main application of our results.

Corollary 8.6. The $\omega$-inequality problem is decidable for all members of the Straubing-Thérien hierarchy.

Acknowledgments. The first author was partially supported by CMUP (UID/MAT/00144/2013), which is funded by FCT (Portugal) with national (MCTES) and European structural funds (FEDER), under the partnership agreement PT2020.

The second and third authors were partially supported by the Grant 1502862S of the Grant Agency of the Czech Republic.

\section{REFERENCES}

1. J. Almeida, Implicit operations on finite $\mathcal{J}$-trivial semigroups and a conjecture of $I$. Simon, J. Pure Appl. Algebra 69 (1990), 205-218.

2. _ Finite semigroups and universal algebra, World Scientific, Singapore, 1995, English translation. 
3. - Hyperdecidable pseudovarieties and the calculation of semidirect products, Int. J. Algebra Comput. 9 (1999), 241-261.

4. $ـ$ Profinite semigroups and applications, Structural theory of automata, semigroups and universal algebra (New York) (V. B. Kudryavtsev and I. G. Rosenberg, eds.), Springer, 2005, pp. 1-45.

5. J. Almeida, J. Bartoňová, O. Klíma, and M. Kunc, On decidability of intermediate levels of concatenation hierarchies, Developments in Language Theory (I. Potapov, ed.), Lect. Notes in Comput. Sci., no. 9168, 2015, pp. 58-70.

6. J. Almeida, A. Cano, O. Klíma, and J.-É. Pin, On fixed points of the lower set operator, Int. J. Algebra Comput. 25 (2015), 259-292.

7. J. Almeida and A. Costa, Infinite-vertex free profinite semigroupoids and symbolic dynamics, J. Pure Appl. Algebra 213 (2009), 605-631.

8. J. Almeida, J. C. Costa, and M. Zeitoun, Iterated periodicity over finite aperiodic semigroups, European J. Combin. 37 (2014), 115-149.

9. —— McCammond's normal forms for free aperiodic semigroups revisited, LMS J. Comput. Math. 18 (2015), 130-147.

10. Comp. Sci. 18 (2016).

11. J. Almeida and B. Steinberg, On the decidability of iterated semidirect products and applications to complexity, Proc. London Math. Soc. 80 (2000), 50-74.

12. S. Burris and H. P. Sankappanavar, A course in universal algebra, Grad. Texts in Math., no. 78, Springer, Berlin, 1981.

13. R. S. Cohen and J. A. Brzozowski, Dot-depth of star-free events, J. Comput. System Sci. 5 (1971), 1-15.

14. S. Eilenberg, Automata, languages and machines, vol. B, Academic Press, New York, 1976.

15. S. J. van Gool and B. Steinberg, Pro-aperiodic monoids via saturated models, Tech. report, 2016, arXiv:1609.07736.

16. H. J. Keisler, Fundamentals of model theory, Handbook of Mathematical Logic (J. Barwise, ed.), Studies in Logic and the Foundations of Mathematics, vol. 90, North Holland, Amsterdam, 1977, pp. 47-104.

17. J. McCammond, Normal forms for free aperiodic semigroups, Int. J. Algebra Comput. 11 (2001), 581-625.

18. J. D. McKnight, Jr. and A. J. Storey, Equidivisible semigroups, J. Algebra 12 (1969), 24-48.

19. V. A. Molchanov, Nonstandard characterization of pseudovarieties, Algebra Universalis 33 (1995), 533-547.

20. J.-E. Pin, Eilenberg's theorem for positive varieties of languages, Russian Math. (Iz. VUZ) 39 (1995), 74-83.

21. _ Syntactic semigroups, Handbook of Formal Languages (G. Rozenberg and A. Salomaa, eds.), Springer, 1997.

22. J.-E. Pin and P. Weil, Profinite semigroups, Mal'cev products and identities, J. Algebra 182 (1996), 604-626.

23. _ A Reiterman theorem for pseudovarieties of finite first-order structures, Algebra Universalis 35 (1996), 577-595.

24. _ Polynomial closure and unambiguous product, Theory Comput. Syst. 30 (1997), 383-422.

25. T. Place and M. Zeitoun, Going higher in the first-order quantifier alternation hierarchy on words, Automata, languages, and programming. Part II (ICALP'14) (J. Esparza, P. Fraigniaud, T. Husfeldt, and E. Koutsoupias, eds.), Lect. Notes in Comput. Sci., vol. 8573, 2014, pp. 342-353.

26. $ـ$ Separating regular languages with first-order logic, CSL-LICS'14, 2014, DOI 10.1145/2603088.2603098.

27. J. Reiterman, The Birkhoff theorem for finite algebras, Algebra Universalis 14 (1982), $1-10$.

28. J. Rhodes and B. Steinberg, The q-theory of finite semigroups, Springer Monographs in Mathematics, Springer, 2009. 
29. H. Straubing, A generalization of the Schützenberger product of finite monoids, Theor. Comp. Sci. 13 (1981), 137-150.

30. $53-94$.

31. W. Thomas, Classifying regular events in symbolic logic, J. Comput. System Sci. 25 (1982), 360-376.

32. D. Thérien, Classification of finite monoids: the language approach, Theor. Comp. Sci. 14 (1981), 195-208.

33. S. Willard, General topology, Addison-Wesley, Reading, Mass., 1970.

CMup, Dep. Matemática, Faculdade de Ciências, Universidade do Porto, Rua do CAmpo Alegre 687, 4169-007 Porto, Portugal

E-mail address: jalmeida@fc.up.pt

Dept. of Mathematics and Statistics, Masaryk University, Kotlár̆ská 2, 61137 Brno, Czech Republic

E-mail address: klima@math.muni.cz, kunc@math.muni.cz 